\title{
Future Benefits of Time-Varying Gravity Missions to Ocean Circulation Studies
}

\author{
Chris W. Hughes ${ }^{1^{\star}}$ and Pascal Legrand ${ }^{2^{\star}}$
}

\author{
${ }^{1}$ Proudman Oceanographic Laboratory, 6 Brownlow Street, Liverpool, L3 5DA, UK \\ ${ }^{2}$ Physical Oceanography Laboratory, IFREMER Brest, BP 70, 29280 Plouzane, France \\ *: Corresponding author : cwh@pol.ac.uk pascal.le.grand@ifremer.fr
}

\begin{abstract}
A summary is offered of the potential benefits of future measurements of temporal variations in gravity for the understanding of ocean dynamics. Two types of process, and corresponding amplitudes are discussed: ocean basin scale pressure changes, with a corresponding amplitude of order $1 \mathrm{~cm}$ of water, or $1 \mathrm{~mm}$ of geoid height, and changes in along-slope pressure gradient, at cross-slope length scales corresponding to topographic slopes, with a corresponding amplitude of order $1 \mathrm{~mm}$ of water, or a maximum of about $0.01 \mathrm{~mm}$ of geoid. The former is feasible with current technology and would provide unprecedented information about abyssal ocean dynamics associated with heat transport and climate. The latter would be a considerable challenge to any foreseeable technology, but would provide an exceptionally clear, quantitative window on the dynamics of abyssal ocean currents, and strong constraints on ocean models. Both options would be limited by the aliassing effect of rapid mass movements in the earth system, and it is recommended that any future mission take this error source explicitly into account at the design stage. For basinscale oceanography this might involve a higher orbit than GRACE or GOCE, and the advantages of exact-repeat orbits and multiple missions should be considered.
\end{abstract}

Keywords: Abyssal - ocean circulation changes - ocean bottom pressure - satellite gravity 


\title{
Future benefits of Time-varying gravity missions to ocean circulation studies
}

\author{
Chris W. Hughes (cwh@pol.ac.uk) \\ Proudman Oceanographic Laboratory, 6 Brownlow St, Liverpool L3 5DA, U.K. \\ Pascal LeGrand (pascal.le.grandeifremer.fr) \\ Physical Oceanography Laboratory, IFREMER Brest, BP 70, 29280 Plouzane, France
}

\begin{abstract}
A summary is offered of the potential benefits of future measurements of temporal variations in gravity for the understanding of ocean dynamics. Two types of process, and corresponding amplitudes are discussed: ocean basin scale pressure changes, with a corresponding amplitude of order $1 \mathrm{~cm}$ of water, or $1 \mathrm{~mm}$ of geoid height, and changes in along-slope pressure gradient, at cross-slope length scales corresponding to topographic slopes, with a corresponding amplitude of order $1 \mathrm{~mm}$ of water, or a maximum of about $0.01 \mathrm{~mm}$ of geoid. The former is feasible with current technology and would provide unprecedented information about abyssal ocean dynamics associated with heat transport and climate. The latter would be a considerable challenge to any foreseeable technology, but would provide an exceptionally clear, quantitative window on the dynamics of abyssal ocean currents, and strong constraints on ocean models. Both options would be limited by the aliassing effect of rapid mass movements in the earth system, and it is recommended that any future mission take this error source explicitly into account at the design stage. For basin-scale oceanography this might involve a higher orbit than GRACE or GOCE, and the advantages of exact-repeat orbits and multiple missions should be considered.
\end{abstract}

\section{Introduction}

Time-varying gravity is, over the ocean, potentially a measure of ocean bottom pressure (OBP) variations. Such variations have been studied from in-situ data predominantly at short (days to months) time scales and at occasional points in the global ocean. The possibility of obtaining multi-year time series of global OBP variations is a very exciting one, and one which is still quite a new concept to the oceanographic community. The first results from GRACE are now beginning to demonstrate this possibility. The future launch of GOCE, which will refine knowledge of the static geoid at smaller length scales, will not significantly address the question of time-dependent gravity relevant to OBP measurement. The purpose of this paper is to look at the possible OBP signals to be measured, for consideration in the design of future systems for measuring time-dependent gravity.

There are two distinct length scales on which OBP variations can be considered: "basin scales" of typically 1000-4000 km, and topographic length scales of about 20-200 km. In what follows, the dynamical information associated with these two length scales is discussed separately.

(C) 2006 Kluwer Academic Publishers. Printed in the Netherlands.

Cwh_astrium_rev2.tex; 5/06/2006; 14:26; p.1 
Currently, however, the theoretical limiting factor on accuracy of OBP determinations obtainable from a GRACE-like mission appears to be the need to correct for high frequency mass movements which are too fast to be measured with the global coverage that is necessary to map their spatial signatures and subtract them from long-period variations. Some thoughts on the requirements of future missions to minimise this problem will also be offered. It is worth noting that, at the time of writing, the accuracy of GRACE data is at least an order of magnitude worse than its theoretical value. No clear reason for this has yet been identified, as the instruments all appear to be performing close to or better than expectations. It is anticipated that significant improvements can be expected as the data processing chain is refined.

\section{Basin scale variability}

On basin scales, it has long been predicted (Gill and Niiler, 1973), and more recently observationally verified (Fukumori et al., 1998), that most of the intraseasonal to seasonal OBP variability is due to the ocean's depth-independant response to atmospheric wind stress and pressure forcing. This is of little intrinsic interest except as a source of aliassing for altimetry and temporal satellite gravity measurements, which cannot resample the same spot frequently enough to remove this, sometimes large (up to $5 \mathrm{~cm}$ of water in the deep ocean), high frequency signal from longer term measurements.

At interannual scales, however, the dynamics become much more interesting. The global heat budget is strongly influenced by the ocean circulation, which supplies almost half of the equator-to-pole heat transport which ameliorates what would otherwise be an enormous temperature range around the planet. In the Atlantic, this heat transport is actually northwards in both hemispheres. Such large heat transports are made possible by the sinking of cold water at high latitudes, and spreading throughout the ocean at depth. There are rather few sites of bottom water formation, in the North Atlantic and close to Antarctica, and this water spreads over the whole globe. From the few direct measurements we have, and from the spreading of tracers advected by the slow, deep flows, we know something about the spreading pathways the water takes. It is also known that there are strong variations in production of dense bottom waters as this tends to happen during extreme weather events and is quite sensitive to ambient ocean conditions. However, very little is known of the variability in the flow once it sinks to the ocean floor, or of variations in the subsequent upwelling. This is because direct measurements of deep flows are difficult to make, and therefore rather sparse. In some cases, deep flows may be detectable indirectly by their influence on near-surface circulations, but this is an indirect representation of the deep flow and may be swamped by other near-surface dynamics, making it difficult to infer the deep flow. 
Movement of dense water between ocean basins must, however, produce a signal in ocean bottom pressure. A simple analytical argument (Hughes and Stepanov, 2003) shows that this signal will be predominantly at basin scales as ocean dynamics will rapidly smooth the pressure along depth contours. There is a strong dynamical constraint on the component of pressure gradient along a depth contour, but no such constraint on the pressure averaged around a closed depth contour. This permits a range of possible "basin scale" pressure signals: signals may be basin scale along a depth contour, but at a relatively narrow scale across depth contours (for short, closed depth contours, such as those around a seamount, both scales can be small), or can be basin scale in both directions. The latter are susceptible to measurement using current capability.

Multi-year time series of bottom pressure data at basin scales, in combination with more routine near-surface measurements, will make it possible to track the motion of dense water through the global ocean, from its source to its still poorly-defined upwelling regions, making a major contribution to monitoring and understanding the ocean-atmosphere climate system. Such a monitoring system would ideally be a part of an ongoing monitoring effort to measure interannual to decadal changes in ocean circulation.

It is difficult to specify the size of the expected signals in advance. Modelling results (Hughes and Stepanov, 2003) suggest typical interannual pressure changes of around $1 \mathrm{~cm}$ of water (equating to about $1 \mathrm{~mm}$ change in geoid at these length scales), but there is now strong evidence that much larger changes can occur. Using Lageos satellite tracking data, Cox and Chao (2002) presented a time series of the $J_{2}$ coefficient of the earth's gravity field. This shows a steady decrease, attributable to the secular relaxation of the earth after melting of ice from the last glacial period, but also shows a sudden jump upwards (of about $10^{10}$ ) in mid 1998. Dickey et al.(2002) attributed about half of this jump to a change in OBP, predominantly in the Southern Ocean. While this attribution depends heavily on data assimilation into a coarse resolution ocean model (which is unlikely to represent the strongly eddying Southern Ocean well, and is limited by a dearth of subsurface data in this region), it is undoubtedly true that there was a major sea level drop of about $15 \mathrm{~cm}$ in the Pacific sector of the Southern Ocean at this time (Fig. 1). If this is associated with a mass change, the associated geoid movement is of order $1 \mathrm{~cm}$.

This drop occurred at the same time as the switch from El Niño to La Niña conditions, as can be seen from the large tropical Pacific sea level changes, and highlights both the possible interest of satellite gravity measurements and also the difficulty in interpreting other forms of data. The sea level change in the Southern Ocean is likely to be associated with a mass anomaly, since Southern Ocean flows tend to penetrate to the ocean floor, although model diagnostics suggest that the relationship between sea level and OBP is ge- 


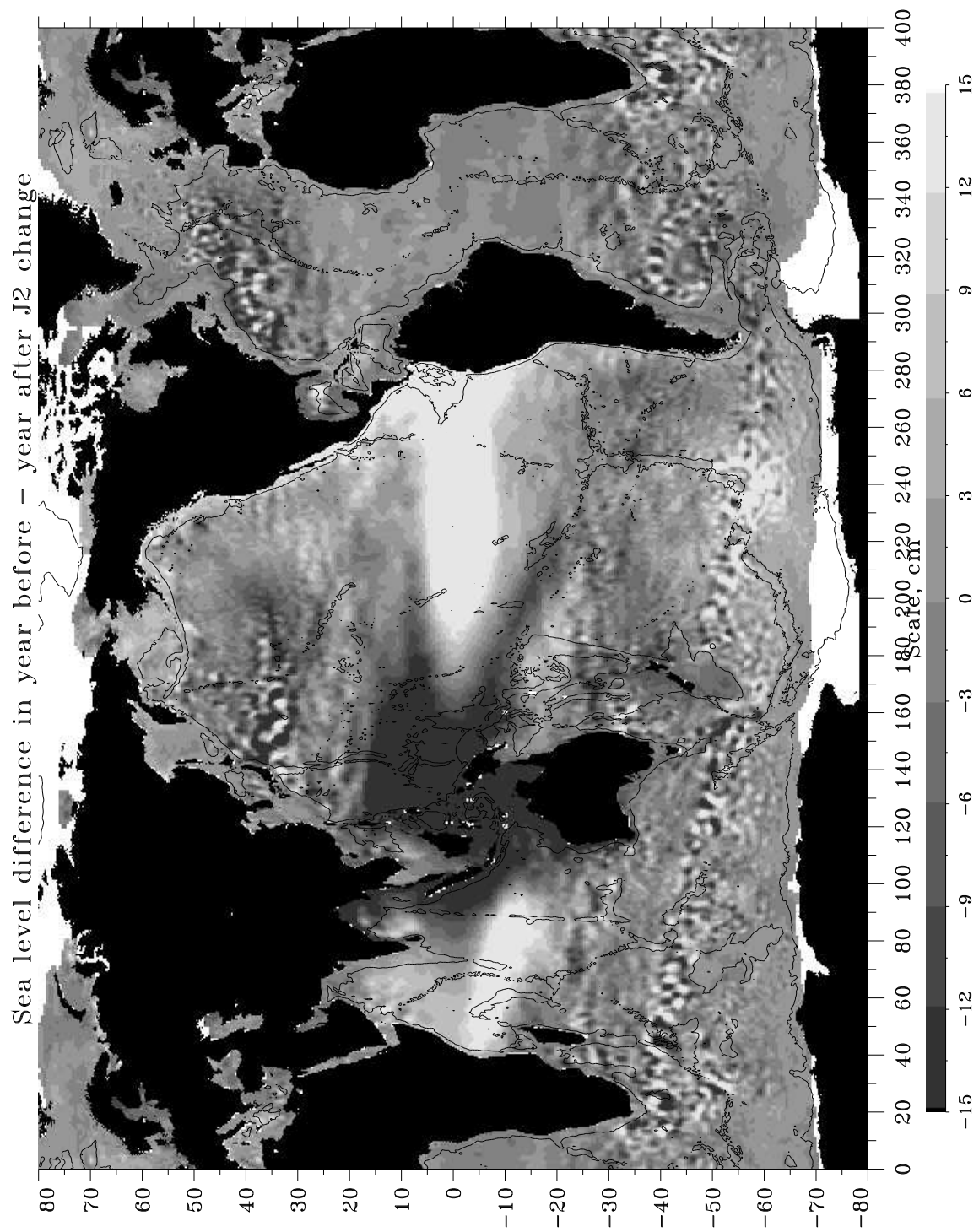

Figure 1. The difference between two annual average sea level fields, year before minus year after the change in $J_{2}$, from satellite altimetry. The $3 \mathrm{~km}$ depth contour is superimposed.

ographically surprisingly variable in the Southern Ocean (R. Bingham, personal communication). In the tropics, however, a sea level rise tends to be strongly affected by heating, and compensated by a depression in the thermocline, producing a much smaller bottom pressure signal. This means that sea level is a poor guide to movements of mass at low latitudes, and cannot tell 
us where the "missing mass" removed from the Southern Ocean has gone. Interpretation of model data is also fraught with difficulties given the small amount of data from the depths of the ocean to constrain the models. It is likely that the sea level change is simply the surface signature of a much larger global redistribution of mass which we cannot see. Even in itself, this $15 \mathrm{~cm}$ sea level change is enough to account for $1=3$ to $1=2$ of the change in $J_{2}$, if we assume the mass removed from the Southern Ocean is redistributed at tropical latitudes.

This is a completely unexpected phenomenon. It is unclear whether its relationship to El Niño is coincidence or something deeper, but it represents a major shift in the ocean in this region. Interpretation would be greatly helped if the mass changes could be more localised, as by a GRACE-type mission. Such a major discovery, associated with the most climatically important and most thoroughly studied ocean phenomenon, shows how interesting even one coefficient of the gravity field can be.

Of equal topical interest is the possibility that the North Atlantic might switch into a different mode of circulation in which much less bottom water is formed, with possibly dramatic consequences for the Northern Hemisphere. Palaeoceanographers argue that such changes occurred during the glacialinterglacial transition (Clark et al., 2002), and there is concern that current anthropogenic climate forcing could tip the system into a state where such a "thermohaline collapse" could occur again. There are several international programmes as part of CLIVAR, whose objective is to monitor the ocean for such changes, but their geographical range is necessarily limited; temporal gravity measurements would be a great help in such monitoring efforts.

The projected accuracy of the current GRACE mission would be easily adequate to resolve these kinds of variability, with an estimated accuracy of $1 \mathrm{~mm}$ of water for a length scale of about $1000 \mathrm{~km}$ (Wahr et al., 1998), although the present actual accuracy appears to be closer to $1 \mathrm{~cm}$ of water at basin scales. However, that is a measure of the instrument accuracy, ignoring the considerable problem of aliassing high frequency variability. If the processing of GRACE data can be improved to boost the system accuracy, the problem of unmodelled tides and barotropic ocean variability particularly in shelf seas and at high latitudes, is likely to become the dominant source of noise (Knudsen, 2003; Ray et al., 2003; Hughes and Stepanov, 2003).

\section{Topographic scale variability}

In order to go further than monitoring basin-scale mass redistributions, a leap in resolution is necessary to permit more detailed attribution of the associated dynamics. It will never be possible to use gravity alone to monitor time- 
averaged flows, but bottom pressure changes can contain information about changes in the currents.

Currents associated with these deep mass transports are typically concentrated against steep topography, with length scales between about 20 and 200 $\mathrm{km}$. A simple estimate of the gravity anomaly associated with subtraction of a uniform $0.2 \mathrm{~kg} / \mathrm{m}^{3}$ density anomaly, occurring over a $1 \mathrm{~km}$ thick layer and $100 \mathrm{~km}$ horizontal extent, produces a geoid anomaly of $1.2 \mathrm{~mm}$. Removal of such a density anomaly is equivalent to a change of about $20 \mathrm{~cm}$ of water, and would represent the complete extinguishing of a major deep western boundary current, as discussed above in the case of the N. Atlantic. This is an extreme upper bound to the size of signal which can be expected, we would expect more usual changes to be at least an order of magnitude smaller. Thus, while movement of dense water between basins can lead to a basinwide signal of a few $\mathrm{cm}$ of water, the fluctuating currents which lead to these transports are associated with changes of similar magnitude, but over smaller length scales, making them correspondingly harder to detect from space.

Going further than this, we can consider the possibility of measuring the much smaller variation of pressure along depth contours. Hughes and de Cuevas (2001) demonstrated the importance of the interaction between bottom pressure and topography for the depth-integrated ocean flow. In principle, if wind stress, topography, and OBP were perfectly known, the depthintegrated flow could be deduced on length scales of about $200 \mathrm{~km}$. As noted before, the absolute value of OBP will never be determined from gravity alone, but fluctuations could in principle be related to fluctuations of the depth-integrated flow, given a reasonable knowledge of the topography. The difficulty lies in the small signals, and small length scales needed: the dynamically significant variable is the gradient of pressure along a depth contour, so pressure needs to be known on the length scale over which topography varies, which can be as small as $10 \mathrm{~km}$. In addition, the strong constraint resulting from this relationship means the associated pressure signals are of order a few $\mathrm{mm}$ of water, even for quite large changes of transport (although the pressure change across depth contours can be much larger). For a $1 \mathrm{~mm}$ change at $50 \mathrm{~km}$ length scale, this would require a geoid accuracy a factor of 400 better than that considered above for a $20 \mathrm{~cm}$ change at $100 \mathrm{~km}$ resulting in the need to measure the geoid to an accuracy of at least $0.03 \mathrm{~mm}$ on length scales of 50 $\mathrm{km}$, or higher for smaller length scales. Benefits to ocean circulation studies are potentially very large, but the technical challenge is also very large.

\section{Accuracy requirements}

As stated in section 2, The projected accuracy of GRACE is sufficient to measure basin scale variations, although current error estimates suggest that in 
practice this capability is marginal. Should the projected accuracy be achieved, the problem would then be with aliassing of high frequency variability. While every effort is being made to model as much of this as possible, it is likely to remain the major error source for the forseeable future. Global barotropic models will certainly develop, and may make a significant impact on storm surge modelling for shelf seas if they reach adequate resolution (and if adequate meteorological forcing data are available). Tides are still a problem, particularly in the Arctic (Ray, 2003), and there are few observations against which to test models.

A good argument can be made for a gravity mission specifically designed to address these problems. There is an analogy in satellite altimetry, in which Geosat demonstrated the potential, but TOPEX/Poseidon first really unlocked that potential by choosing an orbit specifically designed to sample the tides efficiently, thus permitting them to be accurately removed from the signal. Even the relatively straightforward sampling of the ocean by Geosat produced important ambiguities between tidal error signals and long period ocean dynamics. If we think of GRACE as analogous to Geosat, we can use the information gathered by GRACE to help design a mission to minimise such ambiguities.

If basin scale dynamics are the aim, it is possible that the optimum configuration would be an orbit higher than the GRACE orbit, enhancing the spatial averaging capability of each satellite pass and improving the temporal sampling. The spatial resolution would go down in a "no high frequency noise" scenario, but in the real world the useful spatial resolution may improve. An exact repeat orbit would make interpretation of aliasing much simpler, and mutiple instruments could be used to improve the trade-off between temporal and spatial sampling. In addition, if the orbit is designed with sampling of tides in mind, a major source of error could be rapidly reduced.

For topography scale dynamics (say $100 \mathrm{~km}$ ), the requirements are much stricter. The maximum plausible signal is about $1 \mathrm{~mm}$ of geoid, which is beyond the capability of both GOCE and GRACE. However, the principle interest is in ocean signals at long time scales. If technological improvements could improve the GOCE precision from $1 \mathrm{~cm}$ to $0.5 \mathrm{~cm}$ over 6 months, then simple statistical averaging could make $1 \mathrm{~mm}$ a resonable aim for the difference of two 10 year averages. Further improvements in technology (such as superconducting gradiometers and laser tracked satellite constellations) may make fractions of a millimetre a plausible aim, in which case the improved spatial resolution of a low orbit, gradiometry mission may help with the aliassing problem by using the different spatial distributions of the main high frequency "noise" terms from the lower frequency "signal" terms. The problem of resolving topographically-steered boundary current changes is made more difficult by their usual proximity to land, meaning that leakage of land hydrography signals (which can be much larger) will also be a problem. In 
short, resolution of topography-scale dynamics is a big challenge which may just barely be attainable with a major improvement in accuracy.

To go further and diagnose transport fluctuations directly from changes in the gradient of pressure along depth contours would require a further order of magnitude improvement in both measurement accuracy and reduction of aliasing error. Although the dynamical value of such measurements would be high, this does not seem plausible avenue to explore for the foreseeable future.

Whichever option is followed, it seems clear that the mission design is critical. If signal and noise are to be separated, a judicious choice of orbit(s), duration, and measured parameters must be made which takes account not only of instrument noise but also geophysical noise to optimise detection of the desired signal. This would be different for different signals. For example, basin-scale ocean dynamics might best be detected from a slightly higher orbit which would reduce temporal aliasing, but land hydrology has a larger signal and may have a smaller aliasing problem, and might benefit from the lowest orbit possible. As has been found with satellite altimetry, the best exploitation of the technology, minimising aliasing problems, may require multiple gravity missions (and perhaps a mixture of technologies) in order to optimise both the spatial and temporal sampling.

\section{Conclusion}

Important new understanding of how the ocean works will undoubtedly be derived from measurements of time-dependent gravity, as can be seen from the interest already generated by changes in a single coefficient $\left(J_{2}\right)$. The easier aim, which will provide a first global window on ocean thermohaline circulation variations and their role in heat transport, is to measure basin scales of $1000-4000 \mathrm{~km}$, but with an instrument designed to minimise the impact of geophysical noise. Geoid signals of order $1 \mathrm{~mm}$ or more are to be expected, within the range of current technology. Moderate accuracy improvements would increase the spatial resolution attainable and aid the interpretation of the signal, but would not produce a step change in value of the observing system.

The more ambitious aim is to look for resolutions in the range of 20-200 $\mathrm{km}$ capable of collocating pressure anomalies with topographic features. The most gross changes in circulation would produce signals of order $1 \mathrm{~mm}$ in the geoid, while more subtle signals containing important dynamical information would require accuracies of order $0.01 \mathrm{~mm}$ on length scales smaller than 100 $\mathrm{km}$. Should that be achievable, however, the gain for oceanography would be enormous, permitting the depth-integrated flow to be calculated at about $200 \mathrm{~km}$ resolution and giving a clear picture of flows near the ocean floor. 
The current and projected observational network is only capable of providing very limited information on such flows. However, the accuracy needed for the more subtle signal of pressure gradient along topography appears beyond anything currently foreseeable.

Whatever the aim of any future mission, a serious complicating factor which must be take into account is aliasing of high frequency signals. Lessons must be learned from GRACE to help design a mission so as to minimise these errors. The trade-off between temporal and spatial resolution should be carefully examined in the light of the signal to be measured, as should the potential advantages of an exact repeat orbit. The clear interpretation of both ocean and land signals may require multiple missions.

\section{References}

Clark, P. U., N. G. Pisias, T. F. Stocker, and A. J. Weaver, 2002: The role of the thermohaline circulation in abrupt climate change. Nature, 415(6874), 863-869.

Cox, C. M., and B. F. Chao, 2002: Detection of a large-scale mass redistribution in the terrestrial system since 1998. Science, 297, 831-883.

Dickey, J. O., S. L. Marcus, O. de Viron O, and I. Fukumori, 2002: Recent Earth oblateness variations: Unraveling climate and postglacial rebound effects Science, 298, 1975-1977.

Fukumori I, R. Raghunath R, and L. -L. Fu, 1998: Nature of global large-scale sea level variability in relation to atmospheric forcing: A modeling study. J. Geophys. Res., 103, 5493-5512.

Gill, A. E. and P. P. Niiler, 1973: The theory of the seasonal variability in the ocean, Deep-Sea Res., 20, 141-177.

Hughes, C. W., and B. A. de Cuevas, 2001: Why western boundary currents in realistic oceans are inviscid: A link between form stress and bottom pressure torques. J. Phys. Oceanogr., 31, 2871-2885.

Hughes, C. W., and V. Stepanov, 2003: Feasibility, and contribution to ocean circulation studies, of ocean bottom pressure determination. Space Science Reviews, 108, 217-224.

Knudsen, P., 2003: Ocean tides effects in GRACE gravimetry. Space Science Reviews, 108, 261-270.

Ray, R. D., D. D. Rowlands, and G. D. Egbert, 2003: Tidal models in a new era of satellite gravimetry. Space Science Reviews, 108, 271-282.

Wahr J., M. Molenaar, and F. Bryan, 1998: Time variability of the Earth's gravity field: Hydrological and oceanic effects and their possible detection using GRACE. J. Geophys. Res., 103(B12), 30205-30229

Address for Offprints: Chris W. Hughes, Proudman Oceanographic Laboratory, 6 Brownlow St, Liverpool L3 5DA, U.K. (cwh@ pol.ac.uk) 
Cwh_astrium_rev2.tex; 5/06/2006；14:26; p.10 